\title{
Erratum to: Video Analytics
}

\author{
Kamal Nasrollahi ${ }^{1(凶)}$, Cosimo Distante ${ }^{2}$, Gang $\mathrm{Hua}^{3}$, \\ Andrea Cavallaro ${ }^{4}$, Thomas B. Moeslund ${ }^{1}$, \\ Sebastiano Battiato ${ }^{5}$, and Qiang $\mathrm{Ji}^{6}$ \\ 1 Aalborg University, Aalborg, Denmark \\ kn@create.aau.dk \\ ${ }^{2}$ Institute of Applied Sciences and Intelligent Systems, Lecce, Italy \\ 3 Stevens Institute of Technology, Hoboken, NJ, USA \\ ${ }^{4}$ Queen Mary University of London, London, UK \\ 5 Università di Catania, Catania, Italy \\ ${ }^{6}$ Rensselaer Polytechnic Institute, Troy, NY, USA
}

\section{Erratum to: \\ K. Nasrollahi et al. (Eds.): \\ Video Analytics, LNCS, \\ DOI: 10.1007/978-3-319-56687-0}

In the original version of the papers starting on p. 137 and p. 151, the spelling of Sergio Escalera's name was incorrect. The original chapters were corrected.

The updated original online version for these chapters can be found at

DOI: $10.1007 / 978-3-319-56687-0 \_12$

DOI: 10.1007/978-3-319-56687-0_13 